\title{
Nutritional habits and bladder cancer
}

\author{
Giuseppe Lippi ${ }^{1}$, Daniele Del Rio ${ }^{2}$ \\ ${ }^{1}$ Section of Clinical Biochemistry, University of Verona, Verona, Italy; ${ }^{2}$ The Laboratory of Phytochemicals in Physiology and the Microbiome \\ Research Hub, University of Parma, Parma, Italy \\ Correspondence to: Prof. Giuseppe Lippi. Section of Clinical Biochemistry, University Hospital of Verona, Piazzale LA Scuro, 37100 Verona, Italy. \\ Email: giuseppe.lippi@univr.it. \\ Provenance: This is a Guest Editorial commissioned by Section Editor Xiao Li (Department of Urologic Surgery, The Affiliated Cancer Hospital of \\ Jiangsu Province of Nanjing Medical University, Nanjing, China). \\ Comment on: Westhoff E, Wu X, Kiemeney LA, et al. Dietary patterns and risk of recurrence and progression in non-muscle-invasive bladder cancer. \\ Int J Cancer 2018;142:1797-804.
}

Submitted Jan 10, 2018. Accepted for publication Jan 15, 2018.

doi: $10.21037 /$ tau.2018.01.11

View this article at: http://dx.doi.org/10.21037/tau.2018.01.11

It is now universally acknowledged that nutrition has a paramount importance in health and disease (1). Cancer is probably one of the most paradigmatic example of a human pathology whose risk of development and progression may be strongly influenced by different dietary habits, through intricate and multifaceted mechanisms entailing a kaleidoscope of still partially enigmatic interplays among genetic, epigenetic, biochemical and also gut-microbial factors (2).

According to recent statistics, bladder cancer is the fifth more frequent malignancy (i.e., approximately $4.7 \%$ of all cancers) and the seventh cause of mortality for cancer $(2.8 \%$ of all cancer deaths), in both sexes (3). The frequency of this cancer is greater in men (i.e., representing the third more frequent malignancy in the male gender), is the highest in North America and Europe, and its pathogenesis has been convincingly associated with many environmental factors such as cigarette smoking, infections (i.e., schistosomiasis), workplace exposures to aromatic amines (e.g., benzidine and beta-naphthylamine), intake of certain drugs (e.g., pioglitazone) or dietary supplements containing aristolochic acid, prior chemotherapy or radiation therapy, and arsenic exposure (4). Some genetic factors have also been associated with an increased risk of developing bladder cancer, mainly represented by polymorphisms in genes encoding for glutathione S-transferase M1 (GSTM1) and $\mathrm{N}$-acetyltransferase 2 (NAT2) (4).

The relationship between dietary habits and bladder cancer is by far more enigmatic. A critical review of the literature, very recently published by Fankhauser and Mostafid, showed that there may be an association between this malignancy and certain foods such as fruits, vegetables, meat, milk products and oil, but also concluded that no reliable evidence exists that a specific diet may be really effective in lowering bladder cancer risk (5). Piyathilake recently gave a nice overview on dietary factors potentially associated with bladder cancer, and confirmed that no strong evidence exists that specific dietary habits, or supplementation with micronutrients, may be effective in reducing bladder cancer risk (6).

In a recent article, Westhoff et al. provided some valuable insights on the still unclear relationship between nutrition and bladder cancer (7). The authors published the results of a large cohort study based on 595 patients with newly diagnosed non-muscle-invasive bladder cancer, who were followed-up for a median period of 66 months. Notably, patients in the highest tertile of adherence to a Western dietary pattern had a $48 \%$ higher risk of recurrence of bladder cancer (hazard ratio, 1.48; $95 \%$ confidence interval, 1.06-2.06) when compared to patients in the lowest tertile, whilst no other statistically significant associations were found between other dietary habits and the risk of progression or recurrence. The socalled Western diet is especially low in fresh fruits and vegetables, but generally high in saturated fats (i.e., fried foods) and red and processed meat, which are associated with a plethora of malignancies. In particular, convincing associations have been reported between high intake of red 
Table 1 Epidemiologic associations between certain foods and risk of bladder cancer

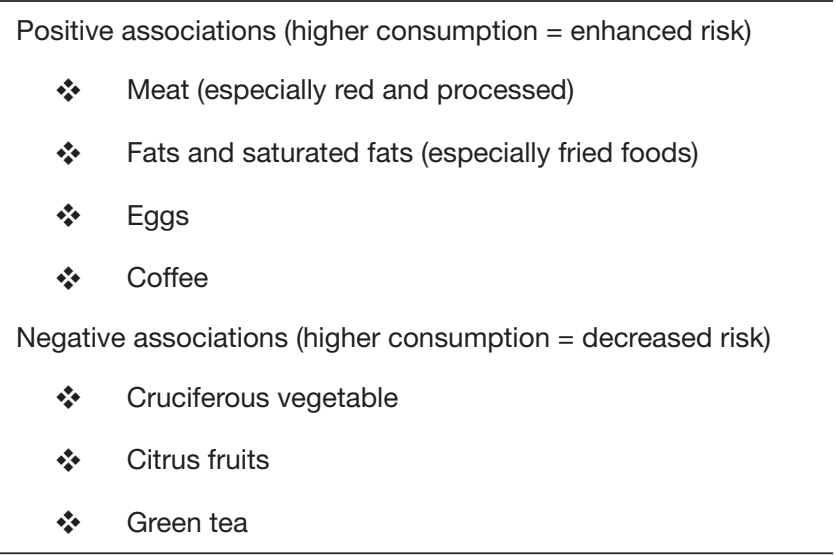

and processed meat with colorectal, lung, esophageal and gastric cancer (8), and also between high consumption of saturated fats (especially fried foods) and prostate, breast, endometrium and ovary cancers $(9,10)$. Notably, a Western diet has also been straightforwardly associated with other, highly prevalent and severe human pathologies, such as cardiovascular disease (11) and diabetes (12), among others.

Albeit the results of the prospective study of Westhoff et al. (7) need to be confirmed in larger prospective investigations, such as the ongoing BLEND (bladder cancer, epidemiology and nutritional determinants) study (13), some clear-cut implications can be drawn. On the assumption that a healthy diet may have a putative impact in reducing the risk of development and progression of bladder cancer, as well as on lowering the risk of developing many other human disorders, a list of foods, whose consumption may be increased or reduced, can be envisaged (Table 1) (6,14-16). Intriguing associations with a reduced bladder cancer risk have also been observed with certain vitamins, especially vitamin $\mathrm{B}, \mathrm{C}, \mathrm{D}$ and $\mathrm{E}$, so that supplementation with these micronutrients may be considered for lowering the overall risk of developing this frequent type of cancer $(6,14-17)$. These general considerations are strengthened by recent evidence that adherence to standard dietary recommendations is quite scarce in bladder cancer survivors (18).

One of the future steps researchers will have to consider in the race to unravel the link between diet and bladder cancer will undoubtedly be built over the urinary tract microbiota. If for years urine has been considered an almost sterile biological fluid, it is now very well known that microorganisms present at urinary tract level have a role in maintaining urinary health (18). Actually, dietary choices may drastically influence the urinary tract microbial ecosystem (19), as, for example, specific urinary metabolites of compounds introduced through a diet rich in plant foods may be bacteriostatic or even show antibiotic activity (20). For this reason, a deeper investigation of the urinary microbiota, and on how it can be influenced by dietary choices, will reasonably lead us to rethink the preventative approaches for bladder cancer.

\section{Acknowledgements}

None.

\section{Footnote}

Conflicts of Interest: The authors have no conflicts of interest to declare.

\section{References}

1. Krehl WA. The role of nutrition in maintaining health and preventing disease. Health Values 1983;7:9-13.

2. Mayne ST, Playdon MC, Rock CL. Diet, nutrition, and cancer: past, present and future. Nat Rev Clin Oncol 2016;13:504-15.

3. Siegel RL, Miller KD, Jemal A. Cancer Statistics, 2017. CA Cancer J Clin 2017;67:7-30.

4. Malats N, Real FX. Epidemiology of bladder cancer. Hematol Oncol Clin North Am 2015;29:177-89, vii.

5. Fankhauser CD, Mostafid H. Prevention of bladder cancer incidence and recurrence: nutrition and lifestyle. Curr Opin Urol 2018;28:88-92.

6. Piyathilake C. Dietary factors associated with bladder cancer. Investig Clin Urol 2016;57 Suppl 1:S14-25.

7. Westhoff E, Wu X, Kiemeney LA, et al. Dietary patterns and risk of recurrence and progression in non-muscleinvasive bladder cancer. Int J Cancer 2018;142:1797-804.

8. Lippi G, Mattiuzzi C, Cervellin G. Meat consumption and cancer risk: a critical review of published meta-analyses. Crit Rev Oncol Hematol 2016;97:1-14.

9. Knekt P, Steineck G, Järvinen R, et al. Intake of fried meat and risk of cancer: a follow-up study in Finland. Int J Cancer 1994;59:756-60.

10. Lippi G, Mattiuzzi C. Fried food and prostate cancer risk: systematic review and meta-analysis. Int J Food Sci Nutr 2015;66:587-9.

11. Anand SS, Hawkes C, de Souza RJ, et al. Food Consumption and its Impact on Cardiovascular Disease: Importance 
of Solutions Focused on the Globalized Food System: A Report From the Workshop Convened by the World Heart Federation. J Am Coll Cardiol 2015;66:1590-614.

12. van Dam RM, Rimm EB, Willett WC, et al. Dietary patterns and risk for type 2 diabetes mellitus in U.S. men. Ann Intern Med 2002;136:201-9

13. Goossens ME, Isa F, Brinkman M, et al. International pooled study on diet and bladder cancer: the bladder cancer, epidemiology and nutritional determinants (BLEND) study: design and baseline characteristics. Arch Public Health 2016 6;74:30.

14. Silberstein JL, Parsons JK. Evidence-based principles of bladder cancer and diet. Urology 2010;75:340-6.

15. Al-Zalabani AH, Stewart KF, Wesselius A, et al. Modifiable risk factors for the prevention of bladder cancer: a systematic review of meta-analyses. Eur J Epidemiol 2016;31:811-51.

16. Liang S, Lv G, Chen W, et al. Citrus fruit intake and

Cite this article as: Lippi G, Del Rio D. Nutritional habits and bladder cancer. Transl Androl Urol 2018;7(Suppl 1):S90-S92. doi: 10.21037/tau.2018.01.11 bladder cancer risk: a meta-analysis of observational studies. Int J Food Sci Nutr 2014;65:893-8

17. He H, Shui B. Folate intake and risk of bladder cancer: a meta-analysis of epidemiological studies. Int J Food Sci Nutr 2014;65:286-92.

18. Longo TA, Gopalakrishna A, Fantony JJ, et al. Effect of diet on bladder cancer survivors. J Clin Oncol 2016;34:abstr 437.

19. Whiteside SA, Razvi H, Dave S, et al. The microbiome of the urinary tract--a role beyond infection. Nat Rev Urol 2015;12:81-90.

20. Mena P, González de Llano D, Brindani N, et al. 5 -( $3^{\prime}, 4^{\prime}$-Dihydroxyphenyl)- $\gamma$-valerolactone and its sulphate conjugates, representative circulating metabolites of flavan3-ols, exhibit anti-adhesive activity against uropathogenic Escherichia coli in bladder epithelial cells. J Funct Foods 2017;29:275-80. 